\title{
SELF-DIFFUSION BEYOND FICK'S LAW
}

\author{
I.M. de SCHEPPER and M.H. ERNST \\ Institute for Theoretical Physics, University of Utrecht, The Netherlands
}

Received 9 April 1979

The Van Hove self-correlation function, the intermediate incoherent scattering function and its Laplace transform are determined asymptotically for a one component fluid in equilibrium, using the mode coupling theory. The results reproduce in the hydrodynamic limit the predictions from Fick's law. The corrections to Fick's law are consistent with a long time tail in the velocity correlation function and with a diverging super Burnett coefficient in the linear diffusion equation.

\section{Fick's law and its extensions}

The process of self-diffusion of a tagged particle in a three dimensional classical fluid of identical particles in equilibrium is as a first approximation well described by Fick's law. It has been shown in the literature ${ }^{1-4}$ ) that extensions of Fick's law involving higher order gradients of the tagged particle density do not exist. In particular the super Burnett coefficient which is the proportionality coefficient of the $\nabla^{4}$-term in a generalized diffusion equation is infinite. This result has been proven on the basis of mode coupling theories ${ }^{1-3}$ ) as well as from the kinetic theory of hard spheres ${ }^{3}$ ).

It is not immediately clear in what sense Fick's law is a first approximation and in which manner it can be extended. Zwanzig ${ }^{5}$ ) has introduced the concept of hydrodynamic limit and showed that in that limit the predictions from Fick's law could possibly be exact. For three dimensional systems the mode coupling theory and the kinetic theory of hard spheres are consistent with this expectation.

Zwanzig's ideas are used here to extend the Fick's law predictions. The correction terms are explicitly calculated using the mode coupling theory. The inconsistency which arises in introducing higher order diffusion coefficients, such as the super Burnett coefficient, in a generalized diffusion equation will be avoided.

The quantity of interest in this paper is the Van Hove self-correlation function

$$
G(r, t)=\left\langle\delta\left(\Delta r_{1}(t)-r\right)\right\rangle,
$$


which expresses for a system in total equilibrium the probability of finding a tagged particle (chosen to be number 1) at time $t$ at the position $r$ when it was initially $(t=0)$ at the position 0 . The microscopic displacement is $\Delta \boldsymbol{r}_{1}(t)=$ $r_{1}(t)-r_{1}(0)$ and the brackets in (1.1) denote a canonical ensemble average at temperature $T\left(\beta=\left(k_{\mathrm{B}} T\right)^{-1}\right)$ and number density $n=N / V$ where each particle has mass $m$. It is assumed that the thermodynamic limit in (1.1) has been taken.

We also consider the intermediate incoherent scattering function which is the spatial Fourier transform of $G(r, t)$,

$$
\begin{aligned}
\tilde{G}(\boldsymbol{k}, t) & =\int \mathrm{d} \boldsymbol{r} \mathrm{e}^{-\mathrm{i} \boldsymbol{k} \cdot r} G(\boldsymbol{r}, t) \\
& =\left\langle\mathrm{e}^{-\mathrm{i} \boldsymbol{k} \cdot \Delta r_{1}(t)}\right\rangle
\end{aligned}
$$

and the Laplace transform of $\tilde{G}(k, t)$ with respect to time

$$
\begin{aligned}
\tilde{\tilde{G}}(\boldsymbol{k}, z) & =\int_{0}^{\infty} \mathrm{d} t \mathrm{e}^{-z t} G(\boldsymbol{k}, t) \\
& =\frac{1}{z+k^{2} \tilde{\tilde{U}}(\boldsymbol{k}, z)} .
\end{aligned}
$$

Here we have introduced the wave number and frequency dependent "diffusion coefficient" $\tilde{\tilde{U}}(\boldsymbol{k}, z)$. With the help of the projection operator formalism ${ }^{6}$ ) it can be expressed as

$$
\tilde{\tilde{U}}(\boldsymbol{k}, z)=\left\langle j_{k}^{*}(z-\hat{L})^{-1} j_{k}\right\rangle,
$$

where $j_{k}=\exp \left(-\mathrm{i} k \cdot r_{1}\right) v_{1} \cdot k / k$, which is the Fourier transform of the taggedparticle current density, the asterisk denotes complex conjugation and $\hat{L}=$ $P_{\perp} L P_{\perp}$, where $L$ is the Liouville operator and $P_{\perp}$ projects orthogonal to the function $\exp \left(-\mathrm{i} k \cdot r_{1}\right)$.

As a first approximation the quantities $G(r, t), \tilde{G}(k, t)$ and $\tilde{\tilde{G}}(\boldsymbol{k}, z)$ are described by the hydrodynamic equation relevant for self-diffusion, i.e. Fick's law. It can be stated in the following equivalent forms

$$
\begin{aligned}
& \frac{\partial}{\partial t} G(r, t) \simeq D \nabla^{2} G(r, t), \\
& \frac{\partial}{\partial t} \tilde{G}(k, t) \simeq-D k^{2} \tilde{G}(k, t), \\
& \tilde{G}(k, z) \simeq \frac{1}{z+D k^{2}} .
\end{aligned}
$$


Here $D$ is the coefficient of self-diffusion in Fick's law. The solution of (1.5) and (1.6) is

$$
\begin{aligned}
& G(r, t) \simeq(4 \pi D t)^{-3 / 2} \mathrm{e}^{-r^{2} /(4 D t)}, \\
& \tilde{G}(k, t) \simeq \mathrm{e}^{-D k^{2} t} .
\end{aligned}
$$

The relations (1.5)-(1.9) are assumed to be approximately valid if distances (c.q. times) are large compared to microscopic lengths (c.q. times) and wave vectors (c.q. frequencies) small compared to inverse microscopic lengths (c.q. inverse microscopic times).

The concept of hydrodynamic limit is used to show in what sense the predictions (1.5)-(1.9) can possibly be exact relations. We illustrate this concept by means of Einstein's displacement formula, stating that the mean square displacement of the tagged particle in the $x$ direction is proportional to $t$ for large times, i.e. $\left\langle\left(\Delta x_{1}(t)\right)^{2}\right\rangle=2 D t$. In fact this relation is a consequence of Fick's law as it is the second moment of $G(r, t)$ in (1.8). Einstein's formula implies that, for large times, the displacement measured in units $\sqrt{D t}$ is a dimensionless quantity of order 1 , namely $\left\langle\left(\Delta x_{1}(t) / \sqrt{D t}\right)^{2}\right\rangle \simeq 2$. This observation suggests to measure typical distances in the diffusion problem in units $\sqrt{D t}$, and one expects these scaled lengths to be of order 1 for large times. We, therefore, study for large times $G(r, t)$ as function of the scaled distance $\rho=r / \sqrt{D t}$ and of the time $t$, where $\rho$ is considered to be of order 1 and $t$ is considered to be large. If for a function of length and time, the limit $t \rightarrow \infty$ is taken at fixed values of the scaled length $\rho$, this limit is referred to as the hydrodynamic limit. The expectation, as expressed by Zwanzig, is that Fick's law may be true exactly in this hydrodynamic limit.

Let us apply these ideas to the functions $G(r, t), \tilde{G}(\boldsymbol{k}, t)$ and $\tilde{\tilde{G}}(\boldsymbol{k}, z)$. We consider $G(r, t)$ as a function of $\rho$ and $t$. For finite values of $\rho$ and large $t$ one sees from (1.8) that $G(\rho \sqrt{D t}, t)=(4 \pi D t)^{-3 / 2} \exp \left(-\rho^{2} / 4\right)$. The function $G(r, t)$ is said to satisfy Fick's law if

$$
\lim _{\substack{t \rightarrow \infty \\ \rho \text { fixed }}}(4 \pi D t)^{3 / 2} G(\rho \sqrt{D t}, t)=\exp \left(-\rho^{2} / 4\right)
$$

is an exact relation. Next we consider the function $\tilde{G}(k, t)$ in the hydrodynamic limit. Since $\tilde{G}(k, t)$ is the Fourier transform of $G(r, t)$ we study this function, for large $t$, as function of dimensionless scaled wave number $\kappa=k \sqrt{D t}$, where $1 / t$ is considered as the small parameter. Inversely we may consider $\tilde{G}(k, t)$ as a function of a dimensionless scaled time $\tau\left(\tau=D k^{2} t\right)$, while $k$ is used as the small parameter. From (1.9) follows then that $\tilde{G}\left(k, \tau / D k^{2}\right) \simeq \exp (-\tau)$ for finite values of $\tau$ and small values of $k$. Now, 
$\tilde{G}(k, t)$ satisfies Fick's law, if

$$
\lim _{\substack{k \rightarrow 0 \\ \tau \text { fixed }}} \tilde{G}\left(\boldsymbol{k}, \tau / D k^{2}\right)=\exp (-\tau) .
$$

Since $\tilde{\tilde{G}}(\boldsymbol{k}, z)$ is the Laplace transform of $\tilde{G}(k, t)$, we measure the frequency in units $D k^{2}\left(z=\zeta D k^{2}\right)$ and study the function $\tilde{\tilde{G}}(k, z)$ as a function of $\zeta$, while $k$ is used as the small parameter. For finite $\zeta$ and $k$ small, Fick's law predicts $D k^{2} \tilde{\tilde{G}}\left(k, \zeta D k^{2}\right) \simeq(\zeta+1)^{-1}$. Hence, $\tilde{\tilde{G}}(k, z)$ satisfies Fick's law, if

$$
\lim _{\substack{k \rightarrow 0 \\ \operatorname{Re} \zeta>0}} D k^{2} G\left(k, \zeta D k^{2}\right)=\frac{1}{\zeta+1} .
$$

In this relation we excluded $\operatorname{Re} \zeta \leqq 0$, since $G(k, z)$ is only defined for values of $z$ with $\operatorname{Re} z>0$. (It may of course be continued analytically into $\operatorname{Re} z \leqq 0$.)

The expressions (1.10)-(1.12) contain the diffusion coefficient $D$. If $G(r, t)$, $\tilde{G}(k, t)$ and $\tilde{G}(k, z)$ satisfy Fick's law, $D$ must be equal to

$$
\lim _{\substack{k \rightarrow 0 \\ \operatorname{Re} z / k^{2} \text { fixed }>0}} \tilde{U}(k, z)=D
$$

for any chosen value of $z / k^{2}$. This statement is a consequence of the exact relation (1.3).

We assume in this paper that the relations (1.10)-(1.13) are valid for systems in three dimensions.

The ansatz that $G(r, t), \tilde{G}(k, t)$ and $\tilde{\tilde{G}}(\boldsymbol{k}, z)$ are essentially functions of a scaled quantity of order 1 and a small ordering parameter will be used here to extend the Fick's law predictions (1.10)-(1.13) beyond the lowest order terms. The small parameters are used systematically as expansion parameters. This means that e.g. $\tilde{G}(k, t)$ is expressed as a power series in $k$ with coefficients which are functions of $\tau=D k^{2} t$, the first term being Fick's law (1.11). Similar expansions will be derived for $G(\boldsymbol{r}, t)$ and $\tilde{\tilde{G}}(\boldsymbol{k}, z)$ and for $\tilde{U}(\boldsymbol{k}, z), \tilde{U}(\boldsymbol{k}, t)$, which is the inverse Laplace transform of $\tilde{\tilde{U}}(k, z)$ and its inverse Fourier transform $U(\boldsymbol{r}, t)$.

In section 2 we review the mode coupling theory and show that it allows expansions of the proposed form. The explicit calculations will be performed in section 3. The predictions for the moments of displacement are given in section 4.

\section{Review of the mode coupling theory}

The mode coupling theory states that for large times $t$ and small wave 
numbers $k$ the correlation function $\tilde{U}(\boldsymbol{k}, t)$ is given by $\left.{ }^{7,8}\right)$

$$
\tilde{U}(k, t) \simeq \int^{\prime} \mathrm{d} q(2 \pi)^{-3} \sum_{\lambda} S^{\lambda}(q, l) \tilde{G}^{\lambda}(q, t) \tilde{G}(l, t),
$$

where $\tilde{U}(\boldsymbol{k}, t)$ is the inverse Laplace transform of the generalized diffusion coefficient $\tilde{U}(\boldsymbol{k}, z)(1.4)$. Here $\boldsymbol{l}=\boldsymbol{k}-\boldsymbol{q}$ and the prime on the integration sign indicates, here and in the following, that $q<k_{0}$. In a dilute gas $k_{0}^{-1}$ is of the order of the mean free path, in a liquid it is of the order of the range of the intermolecular forces. The parameter $\lambda$ in (2.1) runs over the five hydrodynamic modes of the fluid: the heat mode $(T)$, two opposite sound modes $(\sigma= \pm)$ and two shear modes $\left(\eta_{1}, \eta_{2}\right)$, the functions $\tilde{G}^{\lambda}(q, t)$ represent the corresponding hydrodynamic propagators, to which we will return later on. The diffusive propagator $\tilde{G}(l, t)$ is itself defined in terms of the Laplace transform of $\tilde{U}(l, t)$ via (1.3). The quantities $S^{\lambda}$ are to lowest order in $q$ and $l$ given by ${ }^{7,8}$ )

$$
\begin{aligned}
& S^{\eta}(\boldsymbol{q}, l)=\sum_{i=1,2} S^{\eta_{l}}(\boldsymbol{q}, \boldsymbol{l})=(\beta m n)^{-1}(1-\hat{\boldsymbol{k}} \cdot \hat{\boldsymbol{q}})^{2}, \\
& S^{\sigma}(\boldsymbol{q}, \boldsymbol{l})=(2 \beta m n)^{-1}(\hat{\boldsymbol{k}} \cdot \hat{\boldsymbol{q}})^{2},
\end{aligned}
$$

where $\hat{k}$ and $\hat{q}$ denote unit vectors in the $\boldsymbol{k}$ and $q$-direction. The function $S^{T}$ is vanishing to lowest order in $q$ and $l$.

The mode coupling equation (2.1) is supposed to be approximately valid for small wave numbers, $k \ll k_{0}$ and large times $t \gg t_{0}$, where $t_{0}$ is the average time a particle needs to traverse the distance $k_{0}^{-1}$.

Even if the $\tilde{G}^{\lambda}(k, t)$ are given, the set of equations (1.3) and (2.1) is not a closed set for $\tilde{U}(k, t)$ and $\tilde{G}(k, t)$, since the Laplace transform $\tilde{\tilde{U}}(\boldsymbol{k}, z)$ involves also the short time behaviour of $\tilde{U}(k, t)$. The mode coupling theory assumes that this short time behaviour is given by a bare correlation function, which decays fast for all values of $k$. For the long times of interest $\left(t \gg t_{0}\right)$ it can essentially be described as a delta function in time $\delta(t)$ with a coefficient $D^{*}$ independent of $k$. We therefore have for the Laplace transform of $\tilde{U}(k, t)$

$$
\tilde{\tilde{U}}(\boldsymbol{k}, z)=D^{*}+\int_{0}^{\infty} \mathrm{d} t \mathrm{e}^{-z t}(2 \pi)^{-3} \int^{\prime} \mathrm{d} \boldsymbol{q} \sum_{\lambda} S^{\lambda}(\hat{\boldsymbol{q}}, \hat{l}) \tilde{G}^{\lambda}(\boldsymbol{q}, t) \tilde{G}(\boldsymbol{l}, t),
$$

which is only valid for values of $z$ with $|z| \ll z_{0}=t_{0}^{-1}$, and $k \ll k_{0}$. The constant $D^{*}$ is determined by the requirement $\tilde{U}(0,0)=D$, the Fick's law diffusion constant. As discussed in section 1, we are interested in the solutions of the mode coupling equations in the region, where $k$ approaches zero and $t$ approaches infinity, such that $k^{2} t$ remains finite; or equivalently in Laplace language in the region, where $z$ and $k$ approach zero such that $z / k^{2}$ is finite. 
Our basic assumption is that the mode coupling equations describe the dominant singularities in the correlation functions $\tilde{U}(\boldsymbol{k}, z), \tilde{U}(\boldsymbol{k}, t)$ and the hydrodynamic propagators $\tilde{\tilde{G}}(k, z), \tilde{G}(k, t)$ for $k \rightarrow 0$ and $z \rightarrow 0$ with $z /\left(D k^{2}\right)=\zeta$ is finite, or $k \rightarrow 0$ and $t \rightarrow \infty$ with $D k^{2} t=\tau$ is finite.

Before developing a systematic solution to these equations we make a preliminary investigation. Assume according to (1.13) that when $k$ approaches zero $\tilde{U}\left(\boldsymbol{k}, a k^{2}\right)$ approaches a constant independent of $a$ which then, hy definition, is equal to the diffusion coefficient $D$. As a first approximation we write according to (1.9) $\tilde{G}(k, t) \simeq \exp \left(-D k^{2} t\right)$. The hydrodynamic propagators of the fluid are in the same approximation given $\left.b y^{7}\right) \tilde{G}^{\eta}(k, t) \simeq \exp \left(-\nu k^{2} t\right)$ and $\tilde{G}^{\sigma}(k, t) \approx \exp \left(-\mathrm{i} \sigma c k t-\frac{1}{2} \Gamma_{\mathrm{s}} k^{2} t\right)$, where $c$ is the speed of sound, $\Gamma_{\mathrm{s}}$ the sound damping constant and $\nu$ the kinematic viscosity, $\nu=\eta / m n$ where $\eta$ is the shear viscosity.

After substitution of these propagators into (2.4) and using (2.2) and (2.3) one finds the following results. $\tilde{U}(k, z)$ is continuous around $k=0, z=0$ and therefore satisfies eq. (1.13). This means that the mode coupling theory is consistent with Fick's law stated in the rigorous sense of eq. (1.13). Furthermore one finds that deviations of that limiting value are of the form $\sim k h_{1}(\zeta)$ for the shear mode contributions in (2.4) and of the form $k^{2} h_{2}(\zeta)$ for the sound mode contributions. Here and in the sequal $h_{1}(\zeta), h_{2}(\zeta), h(\zeta)$ denote functions of $\zeta=z / D k^{2}$.

A systematic expansion for the functions $U$ and $G$ can be obtained as follows. We define $\Delta \tilde{\tilde{U}}(\boldsymbol{k}, z)$ as

$$
\tilde{\tilde{U}}(\boldsymbol{k}, z)=D+\Delta \tilde{\tilde{U}}(\boldsymbol{k}, z) \text {, }
$$

where $D$ is defined according to (1.13).

The preliminary calculation showed that $\Delta \tilde{\tilde{U}}(k, z)$ is a small quantity of order $k h(\zeta)$. By means of $(1.3)$ we expand $\tilde{G}(k, z)$ in powers of the small quantity $\Delta U$, yielding for finite $\zeta=z /\left(D k^{2}\right)$

$$
D k^{2} \tilde{\tilde{G}}\left(k, \zeta D k^{2}\right)=\frac{1}{\zeta+1}-\frac{\Delta \tilde{\tilde{U}}\left(k, \zeta D k^{2}\right)}{D(\zeta+1)^{2}}+\mathcal{O}\left(k^{2} h(\zeta)\right),
$$

where the first term represents Fick's law (1.12). The function $\Delta \tilde{U}$ follows from (2.4) and (2.5)

$$
\begin{aligned}
\Delta \tilde{\tilde{U}}(\boldsymbol{k}, z)= & \int_{0}^{\infty} \mathrm{d} t \mathrm{e}^{-z t} \int^{\prime} \frac{\mathrm{d} q}{(2 \pi)^{3}} S^{\eta}(q, l) \tilde{G}^{\eta}(q, t) \mathrm{e}^{-D t^{2} t} \\
& -\int_{0}^{\infty} \mathrm{d} t \int^{\prime} \frac{\mathrm{d} q}{(2 \pi)^{3}} S^{\eta}(q,-q) \tilde{G}^{\eta}(q, t) \mathrm{e}^{-D k^{2} t}+\mathcal{O}\left(k^{2} h(\zeta)\right) .
\end{aligned}
$$


The second term represents at fixed $\zeta$ the $k=0$ limit of the first term, and must be introduced due to the definition (2.5). In this paper we will not consider the contributions to $\Delta \tilde{\tilde{U}}$ proportional to $k^{2} h(\zeta)$, so that in $(2.4)$ the sound modes may be neglected. In (2.7) we have used that $G(k, t) \simeq$ $\exp \left(-D k^{2} t\right)$ which corresponds to the first term in the expression (2.6). One can verify that the second term in (2.6) yields corrections to $\Delta \tilde{U}$ of order $k^{2} h(\zeta)$.

After carrying out the time integrals in (2.7) the function $\Delta \tilde{U}(k, z)$ is expressed in the Laplace transfórm $\tilde{\tilde{G}}^{\eta}(\boldsymbol{k}, z)$ of $\tilde{G}^{\eta}(\boldsymbol{k}, t)$

$$
\begin{aligned}
\Delta \tilde{\tilde{U}}(k, z)= & \int^{\prime} \frac{\mathrm{d} q}{(2 \pi)^{3}}\left\{S^{\eta}(q, l) \tilde{\tilde{G}}^{\eta}\left(q, z+D l^{2}\right)-S^{\eta}(q,-q) \tilde{\tilde{G}}\left(q, D k^{2}\right)\right\} \\
& +\mathscr{O}\left(k^{2} h(\zeta)\right) .
\end{aligned}
$$

In order to evaluate (2.8) we use the result for $\tilde{\tilde{G}}^{\eta}(k, z)$ obtained from the mode coupling theory applied to the case of the general fluid ${ }^{8}$ )

$$
\tilde{\tilde{G}}^{\eta}(k, z)=\left(z+\nu k^{2}\right)^{-1}+\sum_{n=1}^{\infty} \Delta_{\eta}(n) k^{2+p_{n}}\left(z+\nu k^{2}\right)^{-2}+\mathcal{O}\left(k^{-1} h(\zeta)\right)
$$

valid for small $k$ and $z / k^{2} \sim \zeta$ finite. The quantities $p_{n}$ are rational numbers between zero and one

$$
p_{n}=1-2^{-n} \quad(n=1,2, \ldots) .
$$

Expressions for the coefficients $\Delta_{\eta}(n)$ are given in ref. 8. We quote only the formula for $n=1$

$$
\Delta_{\eta}(1)=\frac{c^{1 / 2}}{77 \pi 2^{1 / 2} \beta m n \Gamma_{\mathrm{s}}^{3 / 2}},
$$

where $\Gamma_{\mathrm{s}}$ is the sound damping constant given by

$$
\Gamma_{\mathrm{s}}=(\gamma-1) \lambda /\left(n c_{p}\right)+((4 / 3) \eta+\zeta) /(m n),
$$

with $\gamma=c_{p} / c_{V}, \lambda$ is the heat conductivity, and $\zeta$ is the bulk viscosity.

Substitution of eq. (2.9) into (2.8) yields the mode coupling prediction for $\Delta \tilde{\tilde{U}}(\boldsymbol{k}, z)$. One verifies that the term of order $k^{-1} h(\zeta)$ in (2.9) will give rise to terms of order $k^{2} h(\zeta)$ in (2.8), so that $\Delta \tilde{\tilde{U}}(k, z)$ follows, up to terms of the form $k^{2} h(\zeta)$. Explicit calculations will be performed in the next section.

\subsection{The functions $\tilde{\tilde{U}}(\boldsymbol{k}, z)$ and $\tilde{\tilde{G}}(\boldsymbol{k}, z)$}

In order to evaluate the integrals $(2.8)$ and (2.9) for $\Delta \tilde{U}$ we introduce the following quantities

$$
\delta=D /(D+\nu),
$$


thus $\delta$ is a real number between zero and one;

$$
k^{*}=4 \pi \beta m n D(D+\nu),
$$

which has the dimension of an inverse microscopic length such as $k_{0}$;

$$
a_{n}=\Delta_{\eta}(n) D^{-1} \delta^{1+p_{n}} \quad(n=1,2, \ldots),
$$

which have the dimension of a length to the power $p_{n}$;

$$
y_{0}(k)=k_{0} /(\delta k),
$$

which is a large dimensionless quantity for the small $k$-values to be considered below. Instead of $\zeta=z / D k^{2}$ it will be more convenient to define the dimensionless frequency $s$ to be

$$
s(k, z)=\delta^{-1}\left(z /\left(D k^{2}\right)+1\right) \text {. }
$$

The $q$-integral in (2.8) is written as an integral over $x=\hat{\boldsymbol{k}} \cdot \hat{\boldsymbol{q}}$ and $y=|q| /(\delta k)$. In this way we arrive at

$$
\Delta \tilde{U}(k, z)=\delta D \frac{k}{k^{*}} u\left(y_{0}, s\right)+\delta D \frac{k}{k^{*}} \sum_{n=1}^{\infty} a_{n} k^{D_{n}} u_{p_{n}}\left(y_{0}, s\right)+\mathcal{O}\left(k^{2} h(s)\right),
$$

with

$$
\begin{aligned}
& u\left(y_{0}, s\right)=\pi^{-1} \int_{0}^{y_{0}} \mathrm{~d} y \int_{-1}^{+1} \mathrm{~d} x\left(1-x^{2}\right)\left\{\frac{y^{2}}{s-2 x y+y^{2}}-1\right\}, \\
& u_{p}\left(y_{0}, s\right)=\pi^{-1} \int_{0}^{y_{0}} \mathrm{~d} y \int_{-1}^{+1} \mathrm{~d} x\left(1-x^{2}\right) y^{p}\left\{\frac{y^{4}}{\left(s-2 x y+y^{2}\right)^{2}}-1\right\} .
\end{aligned}
$$

The functions $u_{p}\left(y_{0}, s\right)$ will be considered for variable values of $p$ with $0<p<1$. The functions $u\left(y_{0}, s\right)$ and $u_{p}\left(y_{0}, s\right)$ can be studied for fixed values of $s$ and $k \rightarrow 0$ (i.e. $y_{0} \rightarrow \infty$ ). One finds straightforwardly from the asymptotic behaviour of the integrand for large $y$

$$
\begin{aligned}
& u\left(y_{0}, s\right)=u(s)+\mathscr{O}\left(h(s) / y_{0}\right), \\
& u_{p}\left(y_{0}, s\right)=u_{p}(s)+\mathscr{O}\left(h(s) / y_{0}\right),
\end{aligned}
$$

where $u(s)$ and $u_{p}(s)$ are given by the integrals (3.7) and (3.8) respectively with $y_{0}$ replaced by infinity. It follows from (3.4) that the correction terms in (3.9) and (3.10) contribute at least to order $k^{2} h(s)$ in (3.6).

The integrals in (3.7) and (3.8) with $y_{0}=\infty$ are carried out in Appendix A with the result

$$
u(s)=-\frac{2}{3} \sqrt{s_{2}} F_{1}\left(\frac{3}{2},-\frac{1}{2} ; \frac{5}{2} ; \frac{1}{s}\right)
$$




$$
\begin{aligned}
= & \frac{1}{4}(s-2) \sqrt{s-1}-\frac{1}{4} s^{2} \tan ^{-1} \frac{1}{\sqrt{s-1}}, \\
u_{p}(s)= & -\frac{(3+p) s^{(1+p) / 2}}{3 \cos (p \pi / 2)}{ }_{2} F_{1}\left(\frac{5+p}{2},-\frac{1+p}{2} ; \frac{5}{2} ; \frac{1}{s}\right) \\
= & \frac{s^{(4+p) / 2}}{2 \cos (p \pi / 2)}\left[\frac{1}{4+p} \sin \left\{(4+p) \tan ^{-1} \frac{1}{\sqrt{s-1}}\right\}\right. \\
& \left.-\frac{1}{2+p} \sin \left\{(2+p) \tan ^{-1} \frac{1}{\sqrt{s-1}}\right\}\right] .
\end{aligned}
$$

Here ${ }_{2} F_{1}(a, b ; c ; z)$ is Gauss' hypergeometric function. The presentation of our results in hypergeometric functions is convenient in view of the inverse Laplace transforms to be carried out below. Properties of the hypergeometric function ${ }_{2} F_{1}$, the confluent hypergeometric function ${ }_{1} F_{1}(a ; b ; z)$ and the generalized hypergeometric functions ${ }_{p} F_{q}\left(a_{1}, \ldots, a_{p} ; b_{1}, \ldots, b_{q} ; z\right)$, which we will need, are given in ref. 9. Integral representations and asymptotic expansions for large values of argument $z$ are given in ref. 10. The result (3.11b) for $u(s)$ has also been obtained in ref. 11 .

The singularities of $u(s)$ and $u_{p}(s)$ with largest $\operatorname{Re} s$ are square root branch points, located in $s=1$, where the functions behave as

$$
\begin{aligned}
& u(s)=-\frac{\pi}{8}-\frac{\pi}{4}(s-1)+(2 / 3)(s-1)^{3 / 2}+\mathcal{O}\left((s-1)^{2}\right) \\
& u_{p}(s)=\frac{3+p}{(2+p)(4+p)} \operatorname{tg}(p \pi / 2)-\sqrt{s-1}+\mathcal{O}(s-1)
\end{aligned}
$$

The branch point $s=1$ corresponds to $z=-(1-\delta) D k^{2}$ in the complex $z$ plane. The functions are made unique by a cut in the complex $s$-plane on the real $s$ axis from $-\infty$ to 1 . The behaviour of $u(s)$ and $u_{p}(s)$ for large values of $s$ is obtained from (3.11a) and (3.12a)

$$
\begin{aligned}
& u(s)=-(2 / 3) s^{1 / 2}\left[1+\mathcal{O}\left(s^{-1}\right)\right] \\
& u_{p}(s)=-\frac{3+p}{3 \cos (p \pi / 2)} s^{(1+p) / 2}\left[1+\mathcal{O}\left(s^{-1}\right)\right] .
\end{aligned}
$$

The generalized diffusion coefficient $\tilde{\tilde{U}}(\boldsymbol{k}, z)$ is now obtained up to order $\boldsymbol{k}^{2}$ (for $z / k^{2}$ finite). The desired series expansion for $\tilde{G}(k, z)$ follows from (2.6) and (3.6). Using the variable $s$ instead of $\zeta=z / D k^{2}$ yields

$$
\tilde{\tilde{G}}(k, z)=\frac{1}{\delta D k^{2} s}\left[1-\frac{k}{k^{*}} \frac{u\left(y_{0}, s\right)}{s}-\frac{k}{k^{*}} \sum_{n=1}^{\infty} a_{n} k^{p_{n}} \frac{u_{p_{n}}\left(y_{0}, s\right)}{s}+O\left(k^{2} h(s)\right)\right]
$$

and the first term represents Fick's law (1.12). 


\subsection{The functions $\tilde{U}(\boldsymbol{k}, t)$ and $\tilde{G}(\boldsymbol{k}, t)$}

We calculate the functions $\Delta \tilde{U}(\boldsymbol{k}, t)$ and $\tilde{G}(\boldsymbol{k}, t)$ from the results of the previous section. As explained in section 1 we measure time in units $k^{-2}$ and define therefore

$$
\tau=D k^{2} t,
$$

thus the functions $\Delta \tilde{U}(\boldsymbol{k}, t)$ and $\tilde{G}(\boldsymbol{k}, t)$ are expressed in the variables $k$ and $\tau$, instead of $k$ and $t$. We start from

$$
\begin{aligned}
& \Delta \tilde{U}(k, t)=\frac{\delta D k^{2}}{2 \pi \mathrm{i}} \mathrm{e}^{-\tau} \int_{\Gamma} \mathrm{d} s \mathrm{e}^{\delta s \tau} \Delta \tilde{U}\left(\boldsymbol{k}, D k^{2}(\delta s-1)\right), \\
& \tilde{G}(\boldsymbol{k}, t)=\frac{\delta D k^{2}}{2 \pi \mathrm{i}} \mathrm{e}^{-\tau} \int_{\Gamma} \mathrm{d} s \mathrm{e}^{\delta s \tau} \tilde{\tilde{G}}\left(\boldsymbol{k}, D k^{2}(\delta s-1)\right) .
\end{aligned}
$$

These expressions reduce to the relations for the inverse Laplace transforms if one substitutes (3.5) for $s$ and (3.18) for $\tau$. The contour $\Gamma$ runs through the complex $s$ plane from $1+\epsilon-\mathrm{i} \infty$ to $1+\epsilon+\mathrm{i} \infty$, with $\epsilon>0$. From (3.6) and (3.17) one finds expansions for $\Delta \tilde{U}(k, t)$ and $\tilde{G}(k, t)$ of the following form

$$
\begin{aligned}
& \Delta \tilde{U}(k, t)=\delta^{2} D^{2} k^{2}\left[\frac{k}{k^{*}} \mathrm{e}^{-\tau}\left\{v\left(y_{0}, \delta \tau\right)+\sum_{n=1}^{\infty} a_{n} k^{p_{n}} v_{p_{n}}\left(y_{0}, \delta \tau\right)\right\}+\mathcal{O}\left(k^{2} h(\tau)\right)\right], \\
& \tilde{G}(k, t)=\mathrm{e}^{-\tau}\left[1-\frac{k}{k^{*}} w\left(y_{0}, \delta \tau\right)-\frac{k}{k^{*}} \sum_{n=1}^{\infty} a_{n} k^{p_{n}} w_{p_{n}}\left(y_{0}, \delta \tau\right)+\mathcal{O}\left(k^{2} h(\tau)\right)\right],
\end{aligned}
$$

where the functions $v, v_{p}, w$ and $w_{p}$ are given by

$$
\begin{aligned}
& v\left(y_{0}, \tau\right)=\frac{1}{2 \pi \mathrm{i}} \int_{\Gamma} \mathrm{d} s \mathrm{e}^{s \tau} u\left(y_{0}, s\right), \\
& v_{p}\left(y_{0}, \tau\right)=\frac{1}{2 \pi \mathrm{i}} \int_{\Gamma} \mathrm{d} s \mathrm{e}^{s \tau} u_{p}\left(y_{0}, s\right), \\
& w\left(y_{0}, \tau\right)=\frac{1}{2 \pi \mathrm{i}} \int_{\Gamma} \mathrm{d} s \mathrm{e}^{s \tau} u\left(y_{0}, s\right) / s^{2}, \\
& w_{p}\left(y_{0}, \tau\right)=\frac{1}{2 \pi \mathrm{i}} \int_{\Gamma} \mathrm{d} s \mathrm{e}^{s \tau} u_{p}\left(y_{0}, s\right) / s^{2} .
\end{aligned}
$$


The integrals (3.23) and (3.24) are evaluated in Appendix A, with the result $\left(y_{0}=k_{0} / \delta k \rightarrow \infty\right.$ and $\left.\tau \neq 0\right)$

$$
\begin{aligned}
& v\left(y_{0}, \tau\right)=v(\tau)+\mathcal{O}\left(\mathrm{e}^{-y_{0}^{2} \tau}\right), \\
& v_{p}\left(y_{0}, \tau\right)=v_{p}(\tau)+\mathscr{O}\left(\mathrm{e}^{-y_{0}^{2} \tau}\right) .
\end{aligned}
$$

The correction terms of order $\exp \left(-y_{0}^{2} \tau\right)$ will not contribute to any term in the series expansion (3.21) in $k$ since they are non-analytic for $k \rightarrow 0$. The functions $v(\tau)$ and $v_{p}(\tau)$ are given by $(\tau \neq 0)$

$$
\begin{aligned}
v(\tau) & =\frac{1}{3 \sqrt{\pi}} \tau^{-3 / 2} F_{1}\left(\frac{3}{2} ; \frac{5}{2} ; \tau\right) \\
& =\frac{1}{2 \sqrt{\pi}} \tau^{-5 / 2} \mathrm{e}^{\tau}\left[1-\tau^{-1 / 2} \mathscr{F}\left(\tau^{1 / 2}\right)\right], \\
v_{p}(\tau) & =\frac{2}{3 \pi} \Gamma\left(\frac{5+p}{2}\right) \tau^{-(3+p) / 2} F_{1}\left(\frac{5+p}{2} ; \frac{5}{2} ; \tau\right) .
\end{aligned}
$$

The expression (3.29b) is derived from (3.29a) using properties of the confluent hypergeometric function ${ }_{1} F_{1}$ and the relation ${ }^{12}$ )

$$
{ }_{1} F_{1}\left(1 ; \frac{3}{2} ;-\tau\right)=\tau^{-1 / 2} \mathscr{F}\left(\tau^{1 / 2}\right),
$$

where $\mathscr{F}(x)$ is Dawson's integral, related to the error function as $\mathscr{F}(x)=$ $\frac{1}{2} \sqrt{\pi} \mathrm{i} \exp \left(-x^{2}\right) \operatorname{erf}(-\mathrm{i} x)$.

The behaviour of $v(\tau)$ and $v_{p}(\tau)$ for small (and for large) values of $\tau$ is obtained from (asymptotic) series expansions of ${ }_{1} F_{1}{ }^{10}$ )

$$
\begin{aligned}
& v(\tau)=\frac{1}{3 \sqrt{\pi}} \tau^{-3 / 2}[1+\mathcal{O}(\tau)] \\
& v_{p}(\tau)=\frac{2}{3 \pi} \Gamma\left(\frac{5+p}{2}\right) \tau^{-(3+p) / 2}[1+\mathcal{O}(\tau)] \\
& v(\tau)=\frac{1}{2 \sqrt{\pi}} \mathrm{e}^{\tau} \tau^{-5 / 2}\left[1+\mathcal{O}\left(\tau^{-1}\right)\right] \\
& v_{p}(\tau)=\frac{1}{2 \sqrt{\pi}} \mathrm{e}^{\tau} \tau^{-3 / 2}\left[1+\mathscr{O}\left(\tau^{-1}\right)\right]
\end{aligned}
$$

It follows from the asymptotic behaviour of $u(s)$ for $s \rightarrow \infty(3.15)$ and $v(\tau)$ for $\tau \rightarrow 0$ (3.32) that $u(s)$ is not the Laplace transform of $v(\tau)$; this means that the limit $y_{0} \rightarrow \infty$ in (3.23) may not be interchanged with the $s$-integral. The same observation can be made for the functions $u_{p}(s)$ and $v_{p}(s)$. 
The asymptotic behaviour of $u(s)$ and $u_{p}(s)$ for large $s$ allows the interchange of the limit $y_{0} \rightarrow \infty$ and the $s$ integral in (3.25) and (3.26). One finds from (3.9) and (3.10) for $y_{0} \rightarrow \infty$

$$
\begin{aligned}
& w\left(y_{0}, \tau\right)=w(\tau)+\mathcal{O}\left(\frac{h(\tau)}{y_{0}}\right), \\
& w_{p}\left(y_{0}, \tau\right)=w_{p}(\tau)+\mathcal{O}\left(\frac{h(\tau)}{y_{0}^{1-p}}\right) .
\end{aligned}
$$

The correction terms contribute to order $\boldsymbol{k}^{2} \boldsymbol{h}(\tau)$ in (3.22). The functions $w(\tau)$ and $w_{p}(\tau)$ are the inverse Laplace transforms of $u(s) / s^{2}$ and $u_{p}(s) / s^{2}$, respectively. Using the representations (3.11a) and (3.12a) for $u(s)$ and $u_{p}(s)$ and eq. 5.21 (1) of ref. 13 yields

$$
\begin{aligned}
w(\tau) & =-\frac{4}{3 \sqrt{ } \pi} \tau^{1 / 2}{ }_{1} F_{1}\left(-\frac{1}{2} ; \frac{5}{2} ; \tau\right) \\
& =\frac{1}{4 \sqrt{\pi}} \tau^{-1 / 2} \mathrm{e}^{\tau}\left[\tau^{-1 / 2} \mathscr{F}\left(\tau^{1 / 2}\right)\left\{4 \tau^{2}-4 \tau-1\right\}-2 \tau+1\right], \\
w_{p}(\tau) & =\frac{-8 \Gamma((5+p) / 2)}{3 \pi\left(1-p^{2}\right)} \tau^{(1-p) / 2}{ }_{2} F_{2}\left(\frac{5+p}{2},-\frac{1+p}{2} ; \frac{3-p}{2}, \frac{5}{2} ; \tau\right) .
\end{aligned}
$$

Eq. (3.38b) is derived from (3.38a) using properties of ${ }_{1} F_{1}$ and the relation (3.31). The behaviour of $w(\tau)$ and $w_{p}(\tau)$ for small and large $\tau$ is given by

$$
\begin{aligned}
& w(\tau)=-\frac{4}{3 \sqrt{\pi}} \tau^{1 / 2}\{1+\mathcal{O}(\tau)\}, \\
& w_{p}(\tau)=-\frac{8 \Gamma((5+p) / 2)}{3 \pi\left(1-p^{2}\right)} \tau^{(1-p) / 2}\{1+\mathcal{O}(\tau)\}, \\
& w(\tau)=\frac{1}{2 \sqrt{\pi}} \mathrm{e}^{\tau} \tau^{-5 / 2}\left\{1+\mathcal{O}\left(\tau^{-1}\right)\right\} \\
& w_{p}(\tau)=\frac{1}{2 \sqrt{\pi}} \mathrm{e}^{\tau} \tau^{-3 / 2}\left\{1+\mathcal{O}\left(\tau^{-1}\right)\right\}
\end{aligned}
$$

The results (3.21) and (3.22) express $\Delta \tilde{U}(k, t)$ and $\tilde{G}(\boldsymbol{k}, t)$ as series expansions in the smallness parameter $k$ while $\tau=D k^{2} t$ is kept fixed. These expansions may equivalently be considered as expansions in the smallness parameters $1 / t$ with coefficients, which are functions of the dimensionless wave number $\kappa$, defined as

$$
\kappa=k \sqrt{D t}
$$


Thus

$$
\begin{aligned}
\Delta \tilde{U}(\boldsymbol{k}, t)= & \frac{\delta^{2} D^{2}}{k^{*}(D t)^{3 / 2}} \kappa^{3} \mathrm{e}^{-\kappa^{2}}\left\{v\left(\delta \kappa^{2}\right)+\sum_{n=1}^{\infty} \frac{a_{n}}{(D t)^{p_{n} / 2}} \kappa^{p_{n}} v_{p_{n}}\left(\delta \kappa^{2}\right)\right\} \\
& +\mathcal{O}\left(h(\kappa) / t^{2}\right), \\
\tilde{G}(\boldsymbol{k}, t)= & \mathrm{e}^{-\kappa^{2}}\left[1-\frac{\kappa}{k^{*}(D t)^{1 / 2}} w\left(\delta \kappa^{2}\right)\right. \\
& \left.-\frac{\kappa}{k^{*}(D t)^{1 / 2}} \sum_{n=1}^{\infty} \frac{a_{n}}{(D t)^{p_{n} / 2}} \kappa^{p_{n}} w_{p_{n}}\left(\delta \kappa^{2}\right)\right]+\mathcal{O}\left(\frac{h(\kappa)}{t}\right),
\end{aligned}
$$

where we have used $(3.27,28)$ and $(3.36,37)$.

\subsection{The functions $U(r, t)$ and $G(r, t)$}

We calculate the functions $\Delta U(r, t)$ and $G(r, t)$, where $G(r, t)$ is defined in (1.1) and $\Delta U(r, t)$ is defined as the inverse Fourier transform of $\Delta \tilde{U}(k, t)$. As explained in section 1 we use the variables $\rho$ and $t$ instead of $r$ and $t$ to describe these functions, where $\rho$ is defined as

$$
\rho=r / \sqrt{D t} .
$$

For fixed values of $\rho$ the inverse time $1 / t$ is used as small parameter, to obtain expansions beyond the lowest order Fick's law prediction (1.10). We start from the relations

$$
\begin{aligned}
& \Delta U(\boldsymbol{r}, t)=(2 \pi)^{-3} \int \mathrm{d} \boldsymbol{k} \mathrm{e}^{\mathrm{i} \boldsymbol{k} \cdot r} \Delta \tilde{U}(\boldsymbol{k}, t), \\
& G(\boldsymbol{r}, t)=(2 \pi)^{-3} \int \mathrm{d} \boldsymbol{k} \mathrm{e}^{\mathrm{i} \boldsymbol{k} \cdot r} \tilde{G}(\boldsymbol{k}, t) .
\end{aligned}
$$

The parameter $\rho$ is substituted in these expressions and the integration variable $k$ is changed into $(x, \hat{k})$ where $x=k \sqrt{D t}$ (compare (3.44), so that $x=\kappa)$.

The series expansions (3.45) for $\Delta \tilde{U}(\boldsymbol{k}, t)$ and (3.46) for $\tilde{G}(\boldsymbol{k}, t)$ are inserted and we arrive at expressions for $G(r, t)$ and $\Delta U(r, t)$ of the final form

$$
\begin{aligned}
G(r, t)= & \frac{e^{-\rho^{2 / 4}}}{(4 \pi D t)^{3 / 2}} \\
& \times\left[1-\frac{1}{k^{*}(D t)^{1 / 2}} g(\rho)-\frac{1}{k^{*}(D t)^{1 / 2}} \sum_{n=1}^{\infty} \frac{a_{n}}{(D t)^{p_{n} / 2}} g_{p_{n}}(\rho)+\mathcal{O}\left(\frac{h(\rho)}{t}\right)\right]
\end{aligned}
$$


and

$$
\Delta U(r, t)=\frac{\delta^{2} \mathrm{e}^{-\rho^{2 / 4}}}{8 \pi^{3 / 2} D k^{*} t^{3}}\left[f(\rho)+\sum_{n=1}^{\infty} \frac{a_{n}}{(D t)^{p_{n} / 2}} f_{p_{n}}(\rho)+\mathcal{O}\left(\frac{h(\rho)}{\sqrt{t}}\right)\right]
$$

The first term in (3.50) represents Fick's law prediction (1.10).

The functions $g(\rho), g_{p}(\rho), f(\rho)$ and $f_{p}(\rho)$ are given by

$$
\begin{aligned}
& g(\rho)=\frac{4 \mathrm{e}^{\rho^{2 / 4}}}{\sqrt{\pi} \rho} \int_{0}^{\infty} \mathrm{d} x \sin (x \rho) x^{2} \mathrm{e}^{-x^{2}} w\left(\delta x^{2}\right), \\
& g_{p}(\rho)=\frac{4 \mathrm{e}^{\rho^{2} / 4}}{\sqrt{\pi} \rho} \int_{0}^{\infty} \mathrm{d} x \sin (x \rho) x^{2+p} \mathrm{e}^{-x^{2}} w_{p}\left(\delta x^{2}\right), \\
& f(\rho)=\frac{4 \mathrm{e}^{\rho^{2} / 4}}{\sqrt{\pi} \rho} \int_{0}^{\infty} \mathrm{d} x \sin (x \rho) x^{4} \mathrm{e}^{-x^{2}} v\left(\delta x^{2}\right), \\
& f_{p}(\rho)=\frac{4 \mathrm{e}^{\rho^{2 / 4}}}{\sqrt{\pi} \rho} \int_{0}^{\infty} \mathrm{d} x \sin (x \rho) x^{4+p} \mathrm{e}^{-x^{2}} v_{p}\left(\delta x^{2}\right) .
\end{aligned}
$$

These functions also depend on the variable $\delta$. The functions $g(\rho)$ and $g_{p}(\rho)$ are calculated in appendix $B$, with the result

$$
\begin{aligned}
g(\rho)= & -2\left\{\frac{1-\delta}{\pi \delta}\right\}^{1 / 2}\left\{{ }_{1} F_{1}\left(-\frac{1}{2} ; \frac{3}{2} ;-\frac{\delta \rho^{2}}{4(1-\delta)}\right)\right. \\
& \left.-(1-\delta)_{1} F_{1}\left(-\frac{3}{2} ; \frac{3}{2} ;-\frac{\delta \rho^{2}}{4(1-\delta)}\right)\right\} \\
g(\rho)= & \frac{1}{4}\left\{\frac{1-\delta}{\pi \delta}\right\}^{1 / 2}\left[\left(1-5 \delta+\frac{1}{2} \delta \rho^{2}\right) \exp \left(-\frac{\delta \rho^{2}}{4(1-\delta)}\right)+\frac{\sqrt{\pi}}{\sqrt{\delta(1-\delta)} \rho}\right. \\
& \left.\times \operatorname{erf}\left(\sqrt{\frac{\delta \rho^{2}}{4(1-\delta)}}\right)\left\{-(1-\delta)(1+3 \delta)+(1-3 \delta) \delta \rho^{2}+\frac{1}{4} \delta^{2} \rho^{4}\right\}\right]
\end{aligned}
$$

Eq. (3.56) is expressed in more elementary functions (3.57) by means of elementary properties of ${ }_{1} F_{1}$ and the relation $\left.{ }^{12}\right) \operatorname{erf}(x)=$ $2 x_{1} F_{1}\left(1 / 2 ; 3 / 2 ;-x^{2}\right) / \sqrt{\pi}$.

For $g_{p}(\rho)$ we obtained in appendix $\mathbf{B}$ a hypergeometric series of two variables, $\delta$ and $\delta \rho^{2} / 4$ respectively, which can also be written as a series 
expansion in powers of $\rho$

$$
\begin{aligned}
g_{p}(\rho)= & -\frac{4 \Gamma((5+p) / 2) \delta^{(1-p) / 2}}{\pi\left(1-p^{2}\right)} \sum_{m=0}^{\infty} \frac{\left(\frac{5+p}{2}\right)_{m}\left(-\frac{1+p}{2}\right)_{m}}{\left(\frac{3-p}{2}\right)_{m}\left(\frac{3}{2}\right)_{m} m !} \\
& \times{ }_{2} F_{1}\left(m+\frac{5+p}{2}, m-\frac{1+p}{2} ; m+\frac{3-p}{2} ; \delta\right)\left\{1-\frac{\rho^{2}}{4 m+6}\right\}\left\{\frac{-\delta \rho^{2}}{4}\right\}^{m},
\end{aligned}
$$

where we used Pochhammer's symbol $(a)_{n}=\Gamma(a+n) / \Gamma(a)$. The functions $f(\rho)$ and $f_{p}(\rho)$ are hypergeometric series in two variables, $\delta$ and $\delta \rho^{2} / 4$, which converge absolutely in the whole complex $\rho$-plane. This result, and asymptotic expansions, can be obtained by the same method as used in appendix B for $g_{p}(\rho)$. We only quote the results

$$
\begin{aligned}
& f(\rho)=\frac{1}{3 \sqrt{\pi}} \delta^{-3 / 2} I\left(\frac{3}{2} ; \rho\right), \\
& f_{p}(\rho)=\frac{2 \Gamma((5+p) / 2)}{3 \pi} \delta^{-(3+p) / 2} I\left(\frac{5+p}{2} ; \rho\right),
\end{aligned}
$$

where $I(a ; \rho)$ can be written as a series in $\rho^{2}$,

$$
I(a ; \rho)=\sum_{n=0}^{\infty}{ }_{2} F_{1}\left(a+n, \frac{3}{2}+n ; \frac{5}{2}+n ; \delta\right) \frac{(a)_{n}}{\left(\frac{5}{2}\right)_{n} n !}\left\{-\frac{\delta \rho^{2}}{4}\right\}^{n} .
$$

The small $\rho$ behaviour of $g(\rho), g_{p}(\rho), f(\rho)$ and $f_{p}(\rho)$ is given by

$$
\begin{aligned}
g(\rho) & =-2 \sqrt{\frac{\delta(1-\delta)}{\pi}}+\mathcal{O}\left(\rho^{2}\right) \\
g_{p}(\rho) & =-\frac{4 \Gamma((5+p) / 2) \delta^{(1-p) / 2}}{\pi\left(1-p^{2}\right)}{ }_{2} F_{1}\left(\frac{5+p}{2},-\frac{1+p}{2} ; \frac{3-p}{2} ; \delta\right)+\mathcal{O}\left(\rho^{2}\right) \\
f(\rho) & =\frac{\delta^{-3 / 2}}{3 \sqrt{\pi}}{ }_{2} F_{1}\left(\frac{3}{2}, \frac{3}{2} ; \frac{5}{2} ; \delta\right)+\mathcal{O}\left(\rho^{2}\right) \\
& =\frac{\delta^{-3}}{\sqrt{\pi}}\left\{\frac{\sqrt{\delta}}{1-\delta}-\sin ^{-1} \sqrt{\delta}\right\}+\mathscr{O}\left(\rho^{2}\right) \\
f_{p}(\rho) & =\frac{2}{3 \pi} \Gamma((5+p) / 2) \delta^{-(3+p) / 2} F_{2}\left(\frac{5+p}{2}, \frac{3}{2} ; \frac{5}{2} ; \delta\right)+\mathcal{O}\left(\rho^{2}\right) .
\end{aligned}
$$

The behaviour of $g(\rho), g_{p}(\rho), f(\rho)$ and $f_{p}(\rho)$ for large values of $\rho$ is given by 
asymptotic series expansions and we quote only the first terms

$$
\begin{aligned}
& g(\rho)=(1 / 16) \delta \rho^{3}\left[1+\mathcal{O}\left(\rho^{-2}\right)\right] \\
& g_{p}(\rho)=\frac{\delta}{2(4+p) \cos (p \pi / 2)} \rho^{3+p}\left[1+\mathcal{O}\left(\rho^{-2}\right)\right] \\
& f(\rho)=2 \delta^{-3} \rho^{-3}\left[1+\mathcal{O}\left(\rho^{-2}\right)\right] \\
& f_{p}(\rho)=-(4 / \pi)(3+p) \Gamma(2+p) \sin (p \pi / 2) \delta^{-4-p} \rho^{-5-p}\left[1+\mathcal{O}\left(\rho^{-2}\right)\right]
\end{aligned}
$$

\section{Moments of displacement and related quantities}

In this section we calculate the predictions from mode coupling theory for the moments of displacements $M^{(n)}(t)$, the cumulants $M_{c}^{(n)}(t)$ and the time dependent diffusion coefficients $D^{(n)}(t)$. These quantities are defined as

$$
M^{(n)}(t)=\left\langle\left(\Delta x_{1}(t)\right)^{n}\right\rangle,
$$

where $\Delta x_{1}(t)=x_{1}(t)-x_{1}(0)$ is the $x$-component of the displacement vector $\Delta r_{1}(t)$. The odd moments vanish due to symmetry properties of the ensemble average. The even moments occur in the expansion of $\tilde{G}(k, t)$ in powers of $k$. From (1.2) follows

$$
\tilde{G}(k, t)=\sum_{n=0}^{\infty} \frac{\left(-k^{2}\right)^{n}}{(2 n) !} M^{(2 n)}(t)
$$

and therefore

$$
M^{(2 n)}(t)=(-)^{n} \frac{(2 n) !}{n !} \lim _{k \rightarrow 0}\left(\frac{\partial}{\partial k^{2}}\right)^{n} \tilde{G}(k, t)
$$

The cumulants $M_{\mathrm{c}}^{(n)}(t)$ are defined for even $n$ by the series expansion

$$
\tilde{G}(k, t) \equiv \exp \left\{\sum_{n=1}^{\infty} \frac{\left(-k^{2}\right)^{n}}{(2 n) !} M_{\mathrm{c}}^{(2 n)}(t)\right\}
$$

therefore one has for $n=1,2, \ldots$

$$
M_{\mathrm{c}}^{(2 n)}(t)=(-)^{n} \frac{(2 n) !}{n !} \lim _{k \rightarrow 0}\left(\frac{\partial}{\partial k^{2}}\right)^{n} \log \tilde{G}(k, t) .
$$

The time dependent diffusion coefficients are defined by the equation

$$
\frac{\partial}{\partial t} \tilde{G}(k, t) \equiv-k^{2}\left\{\sum_{n=0}^{\infty}\left(-k^{2}\right)^{n} D^{(2 n)}(t)\right\} \tilde{G}(k, t) .
$$


From this and (4.4) follows that

$$
D^{(2 n)}(t)=\frac{1}{(2 n+2) !} \frac{\partial}{\partial t} M_{\mathrm{c}}^{(2 n+2)}(t) .
$$

The prediction for $M^{(n)}(t)$ is obtained from (4.3) and the series expansion (3.22) for $\tilde{G}(k, t)$. We choose a fixed time $t$ and define $x=\delta \tau=\delta D k^{2} t$.

One obtains a series expansion for $M^{(2 j)}(t)$ of the form

$$
\begin{aligned}
M^{(2 j)}(t)= & \frac{(2 j) !}{j !}(D t)^{j}\left[1-\frac{m^{(j)}}{k^{*}(D t)^{1 / 2}}\right. \\
& \left.-\sum_{n=1}^{\infty} \frac{a_{n}}{k^{*}(D t)^{\left(1+p_{n}\right) / 2}} m_{p_{n}}^{(j)}+\mathcal{O}\left(\frac{1}{t}\right)\right]
\end{aligned}
$$

valid for $t$ much larger than $t_{0}$ and $j=0,1, \ldots$ The dimensionless quantities $m^{(j)}$ and $m_{p}^{(j)}$ are given by

$$
\begin{aligned}
& m^{(j)}=\delta^{j-1 / 2} \lim _{x \rightarrow 0}\left(-\frac{\partial}{\partial x}\right)^{j} \mathrm{e}^{-x / \delta} x^{1 / 2} w(x), \\
& m_{p}^{(j)}=\delta^{j-(1+p) / 2} \lim _{x \rightarrow 0}\left(-\frac{\partial}{\partial x}\right)^{j} \mathrm{e}^{-x / \delta} x^{(1+p) / 2} w_{p}(x) .
\end{aligned}
$$

By substitution of the explicit expressions (3.38) and (3.39) for $w(x)$ and $w_{p}(x)$ one finds

$$
\begin{aligned}
& m^{(j)}=\frac{4 j \delta^{1 / 2}}{3 \sqrt{\pi}}{ }_{2} F_{1}\left(-j+1,-\frac{1}{2} ; \frac{5}{2} ; \delta\right), \\
& m_{p}^{(j)}=\frac{8 j \Gamma((5+p) / 2) \delta^{(1-p) / 2}}{3 \pi\left(1-p^{2}\right)}{ }_{3} F_{2}\left(-j+1, \frac{5+p}{2},-\frac{1+p}{2} ; \frac{5}{2}, \frac{3-p}{2} ; \delta\right) .
\end{aligned}
$$

In these expressions ${ }_{2} F_{1}(\ldots ; \delta)$ and ${ }_{3} F_{2}(\ldots ; \delta)$ are polynomials in $\delta$ in degree $j-1$.

For $j=0$ we have $m^{(0)}=0$ and $m_{p}^{(0)}=0$, which is consistent with the property $M^{(0)}(t)=1$ for all $t$, as follows from (4.1). For $j=1$, the polynomials ${ }_{2} F_{1}(\ldots ; \delta)$ and ${ }_{3} F_{2}(\ldots ; \delta)$ in $(4.11,12)$ are equal to 1 , so that the second moment of displacement is given by

$$
\begin{aligned}
M^{(2)}(t)= & 2 D t\left[1-\frac{1}{3 \pi^{3 / 2} \beta m n D(D+\nu)^{3 / 2} t^{1 / 2}}-\frac{2}{3 \pi^{2} \beta m n D}\right. \\
& \left.\times \sum_{n=1}^{\infty} \frac{\Gamma\left(\left(5+p_{n}\right) / 2\right) \Delta_{\eta}(n)}{\left(1-p_{n}^{2}\right)(D+\nu)^{\left(5+p_{n} / 2 / 2\right.} t^{\left(1+p_{n}\right) / 2}}+\mathcal{O}\left(\frac{1}{t}\right)\right],
\end{aligned}
$$

where we have used the relations (3.1), (3.2) and (3.3) for $\delta, k^{*}$ and $a_{n}$.

The time dependent diffusion coefficient $D^{(0)}(t)$. defined by (4.6) is related to $M^{(2)}(t)$ by $D^{(0)}(t)=\frac{1}{2}(\partial / \partial t) M^{(2)}(t)$, as follows from (4.7) and the property that 
$M_{\mathrm{c}}^{(2)}(t)=M^{(2)}(t)$. For $t \gg t_{0}$ we find therefore

$$
\begin{aligned}
D^{(0)}(t)= & D\left[1-\frac{1}{6 \pi^{3 / 2} \beta m n D(D+\nu)^{3 / 2} t^{1 / 2}}-\frac{1}{3 \pi^{2} \beta m n D}\right. \\
& \left.\times \sum_{n=1}^{\infty} \frac{\Gamma\left(\left(5+p_{n}\right) / 2\right) \Delta_{\eta}(n)}{\left(1+p_{n}\right)(D+\nu)^{\left(5+p_{n}\right) / 2} t^{\left(1+p_{n}\right) / 2}}+\mathscr{O}\left(\frac{1}{t}\right)\right] .
\end{aligned}
$$

The velocity correlation function $C(t) \equiv\left\langle v_{1 x} v_{1 x}(t)\right\rangle$, where $v_{1 x}$ is the $x$-component of the tagged particle velocity. Using $C(t)=\partial / \partial t D^{(0)}(t)$ yields for $t \gg t_{0}$

$$
\begin{aligned}
C(t)= & \frac{1}{12 \pi^{3 / 2} \beta m n(D+\nu)^{3 / 2}} \frac{1}{t^{3 / 2}} \\
& \times\left\{1+\sum_{n=1}^{\infty} \frac{2 \Gamma\left(\left(5+p_{n}\right) / 2\right) \Delta_{\eta}(n)}{\pi^{1 / 2}(D+\nu)^{\left(2+p_{n}\right) / 2}} \frac{1}{t^{p_{n} / 2}}\right\}+O\left(\frac{1}{t^{2}}\right) .
\end{aligned}
$$

Next we consider the mode coupling prediction for the cumulants $M_{\mathrm{c}}^{(n)}(t)$ defined in (4.4). Choosing a fixed time $t \gg t_{0}$, the result (3.22) for $\tilde{G}(k, t)$ may be substituted in the expression (4.5) for $M_{\mathrm{c}}^{(2 j)}(t)$. For $j=1$ we have the relation $M_{\mathrm{c}}^{(2)}(t)=M^{(2)}(t)$, where the prediction for $M^{(2)}(t)$ is given by (4.13).

Series expansions for the higher cumulants are obtained from (4.5) and (3.22) by writing $\log \left\{\mathrm{e}^{-\tau}(1-\epsilon)\right\}=-\tau-\epsilon+\mathcal{O}\left(\epsilon^{2}\right)$, where $\epsilon$ represents the terms in (3.22), which are at least of order $k$, at fixed values of $\tau$. The result for $t \gg t_{0}$ and $j \geqq 2$ is

$$
\begin{aligned}
M_{\mathrm{c}}^{(2 j)}(t)= & \frac{(2 j) !}{j !}(D t)^{j}\left[-\frac{q^{(j)}}{k^{*}(D t)^{1 / 2}}\right. \\
& \left.-\sum_{n=1}^{\infty} \frac{a_{n}}{k^{*}(D t)^{\left(1+p_{n}\right) / 2}} q_{p_{n}}^{(j)}+\mathcal{O}\left(\frac{1}{t}\right)\right] .
\end{aligned}
$$

The dimensionless functions $q^{(j)}$ and $q_{p}^{(j)}$ are determined from the relations

$$
\begin{aligned}
& q^{(j)}=(-)^{j} \delta^{j-1 / 2} \lim _{x \rightarrow 0}\left(\frac{\partial}{\partial x}\right)^{j} x^{1 / 2} w(x) \\
& q_{p}^{(j)}=(-)^{j} \delta^{j-(1+p) / 2} \lim _{x \rightarrow 0}\left(\frac{\partial}{\partial x}\right)^{j} x^{(1+p) / 2} w_{p}(x) .
\end{aligned}
$$

By substitution of the expressions (3.38) and (3.39) for $w(x)$ and $w_{p}(x)$ one obtains

$$
\begin{aligned}
& q^{(j)}=(-)^{j} \frac{4 j \delta^{j-1 / 2}}{\sqrt{\pi(2 j+1)(2 j-1)(2 j-3)}}, \\
& q_{p}^{(j)}=(-)^{j} \frac{2 j \Gamma(j+(3+p) / 2) \delta^{j-(1+p) / 2}}{\sqrt{\pi(2 j-1-p)(2 j-3-p) \Gamma(j+3 / 2)}} .
\end{aligned}
$$

Expressions for the time dependent diffusion coefficients $D^{(2 j)}(t)$ defined in 
(4.6) are obtained from (4.7) and (4.16). The result for $t \gg t_{0}$ and $j=1,2, \ldots$ is

$$
\begin{aligned}
D^{(2 j)}(t)= & \frac{(-)^{j}}{2 \pi^{3 / 2}(2 j-1)(2 j+3) j !} \frac{D^{2 j}}{\beta m n(D+\nu)^{j+3 / 2}} t^{j-1 / 2} \\
& \times\left[1+\sum_{n=1}^{\infty} \frac{(2 j-1) \Gamma\left(j+\left(5+p_{n}\right) / 2\right)}{\left(2 j-1-p_{n}\right) \Gamma(j+3 / 2)} \frac{\Delta_{\eta}(n)}{(D+\nu)^{1+p_{n} / 2}} t^{-p_{n} / 2}+O\left(t^{-1 / 2}\right)\right] .
\end{aligned}
$$

For $j=1$ we have

$$
\begin{aligned}
D^{(2)}(t)= & -\frac{D^{2}}{10 \pi^{3 / 2} \beta m n(D+\nu)^{5 / 2}} t^{1 / 2} \\
& \times\left[1+\sum_{n=1}^{\infty} \frac{4 \Gamma\left(\left(7+p_{n}\right) / 2\right)}{3 \sqrt{\pi\left(1-p_{n}\right)}} \frac{\Delta_{\eta}(n)}{(D+\nu)^{1+p_{n} / 2}} t^{-p_{n} / 2}+\mathcal{O}\left(t^{-1 / 2}\right)\right] .
\end{aligned}
$$

The function $D^{(2)}(t)$ is known as the time dependent super Burnett coefficient ${ }^{4}$ ).

\section{Discussion}

We derived an expression for the Van Hove self-correlation function $G(r, t)$, as defined in (1.1), using the mode coupling theory. The result (3.50) expresses $G(r, t)$ as a series in the smallness parameter $1 / t$, where the coefficients are functions of the scaled distance $\rho=r / \sqrt{D t}$. The functions $g(\rho)$ and $g_{p}(\rho)$ are given by (3.57) and (3.58). The leading term in (3.50) represents the prediction for $G(r, t)$ according to Fick's law (1.8) and (1.10). The corrections to Fick's law are at fixed $\rho$ of relative order $t^{-1 / 2}, t^{-3 / 4}, t^{-7 / 8}, \ldots$ in time. The infinite series in (3.50) converges for fixed values of $\rho$, and $t$ sufficiently large ${ }^{8}$ ). Our result implies that $G(r, t)$ satisfies Fick's law in the hydrodynamic limit (i.e. $\rho$ fixed, $t \rightarrow \infty$ ). However, for fixed values of $t$, however large, and $r$ tending to infinity the subsequent terms in (3.50) become increasingly larger, as follows from the asymptotic behaviour of $g(\rho)$ and $g_{p}(\rho)$ given in (3.66) and (3.67). This indicates that Fick's law is not valid for fixed but large $t$ and $r \rightarrow \infty$.

The intermediate scattering function $\tilde{G}(\boldsymbol{k}, t)$ is defined in (1.2). The prediction from the mode coupling theory is given in (3.22). This result expresses $\tilde{G}(k, t)$ as a series in the small parameter $k$ where the coefficients are functions of the scaled time $\tau=D k^{2} t$. The functions $w\left(y_{0}, \delta \tau\right)$ and $w_{p}\left(y_{0}, \delta \tau\right)$ are given by (3.36-39), and the ratio $\delta$ is defined in (3.1). Fick's law holds for $\tilde{G}(k, t)$ in the hydrodynamic limit ( $\tau$ fixed, $k \rightarrow 0$ ). It is not valid for finite $k$, however small, and $t \rightarrow \infty$. This follows from the asymptotic behaviour of $w(\tau)$ 
and $w_{p}(\tau)$ given in $(3.42,43)$. It means that $\tilde{G}(\boldsymbol{k}, t)$ does not behave as $\exp \left(-D k^{2} t\right)$ for finite but small $k$ and $t \rightarrow \infty$.

The mode coupling prediction for $\tilde{\tilde{G}}(k, z)$ defined in (1.3) is given in (3.17). The result is a series expansion in $k$ with coefficients which are functions of the scaled frequency $s$, defined in (3.5). The leading term in (3.17) has a pole in the complex $z$-plane located at $z=-D k^{2}$ (Fick's law). The functions $u(s)$ and $u_{p}(s)$ in $(3.9,10)$ have square root branch point singularities, located at $s=1$ corresponding to $z=(\delta-1) D k^{2}$ (see $(3.13,14)$ ). These singularities are to the right of the hydrodynamic pole at $z=-D k^{2}$. It means that for small but fixed values of $k$, the hydrodynamic polc is not the singularity of $\tilde{\tilde{G}}(k, z)$ closest to the origin in the complex $z$-plane. Consequently Fick's law prediction $\tilde{G}(k, t) \sim \exp \left(-D k^{2} t\right)$ is not the dominant behaviour of $\tilde{G}(k, t)$ for large $t$, as already mentioned above. However Fick's law is still valid in the hydrodynamic limit ( $s$ fixed, $k \rightarrow 0$ ).

The mode coupling theory yields an equation for the generalized diffusion coefficient $\tilde{\tilde{U}}(\boldsymbol{k}, z)$ defined in (1.4). The theory predicts $\tilde{U}(\boldsymbol{k}, z)$ to be continuous around $k=0, z=0$ so that Fick's law (1.13) is satisfied. The explicit predictions for $\Delta \tilde{U}(\boldsymbol{k}, z)=\tilde{\tilde{U}}(\boldsymbol{k}, z)-D$, its inverse Laplace transform $\Delta \tilde{U}(\boldsymbol{k}, t)$ and the inverse Fourier transform $\Delta U(\boldsymbol{r}, t)$ are given in (3.6), (3.21) and (3.51), respectively. The structure of the series expansions is similar to that of $\tilde{G}(\boldsymbol{k}, z), \tilde{G}(\boldsymbol{k}, t)$ and $G(\boldsymbol{r}, t)$.

The long time behaviour of the moments of displacement $M^{(n)}(t)$, defined in (4.1), follows from the result for $\tilde{G}(k, t)$ via (4.3). $M^{(n)}(t)$ diverges dominantly as $t^{n / 2}$, as given in (4.8). The result for $M^{(2)}(t)$ implies the series (4.15) for the velocity correlation function $C(t)$. For large times the contributions to $C(t)$ are proportional to $t^{-3 / ?}, t^{-7 / 4}, t^{-15 / 8}, \ldots$ This agrees with results reported in the literature ${ }^{8,14}$ ).

The cumulants of the moments of displacement, $M_{\mathrm{c}}^{(n)}(t)$ are obtained from (4.5). According to (4.16) $M_{\mathrm{c}}^{(n)}(t)$ diverges for $t \rightarrow \infty$ proportional to $t^{n / 2-1 / 2}$.

We also considered the time dependent diffusion coefficients $D^{(n)}(t)$ in the formally exact linear diffusion equation (4.6). The function $D^{(0)}(t)$ converges for large times to Fick's law diffusion coefficient $D$, as follows from (4.14). All the remaining functions $D^{(n)}(t)$ diverge however proportional to $t^{n / 2-1 / 2}$. For large times a linear diffusion equation involving higher order transport coefficients, such as the super Burnett coefficient, does not exist.

We are not aware of experiments accurate enough to verify the validity of the series expansions for $\tilde{\tilde{G}}(k, z), \tilde{G}(k, t), G(r, t)$ beyond the lowest order term. However, the implicit theoretical predictions for the long time behaviour of the velocity correlation function (4.16) and the time dependent super Burnett coefficient (4.23) do agree with results from molecular dynamics experiments ${ }^{4}$. 
The main results given in this paper have been published before as part of a doctoral dissertation ${ }^{15}$ ).

\section{Appendix A}

Here we will calculate the functions $u(s)$ and $u_{p}(s)$ defined in eqs. (3.7-10) and the functions $v(\tau)$ and $v_{p}(\tau)$ defined in eqs. (3.23-28). The integral (3.7) with $y_{0}=\infty$ for $u(s)$ can be transformed into

$$
u(s)=(2 \pi)^{-1} \int_{0}^{1} \mathrm{~d} x\left(1-x^{2}\right) \int_{-\infty}^{+\infty} \mathrm{d} y\left\{\frac{y^{2}}{s-2 x y+y^{2}}+\frac{y^{2}}{s+2 x y+y^{2}}-2\right\} .
$$

The $y$ integral can be performed by contour integration, yielding

$$
\begin{aligned}
u(s) & =\int_{0}^{1} \mathrm{~d} x\left(1-x^{2}\right)\left(2 x^{2}-s\right)\left(s-x^{2}\right)^{-1 / 2} \\
& =\frac{1}{4}(s-2) \sqrt{s-1}-\frac{1}{4} s^{2} \tan ^{-1} \frac{1}{\sqrt{s-1}} .
\end{aligned}
$$

An expression for $u(s)$ in terms of hypergeometric functions can be obtained by substitution of $y=x^{2}$ in (A.1a),

$$
u(s)=s^{1 / 2} \int_{0}^{1} \mathrm{~d} y y^{-1 / 2}(1-y)\left\{-(1-y / s)^{1 / 2}+\frac{1}{2}(1-y / s)^{-1 / 2}\right\} .
$$

From properties of Gauss' hypergeometric functions ${ }^{9}$ ) follows

$$
\begin{aligned}
u(s) & =-(2 / 3) \sqrt{s}\left\{2{ }_{2} F_{1}\left(\frac{1}{2},-\frac{1}{2} ; \frac{5}{2} ; \frac{1}{s}\right)-{ }_{2} F_{1}\left(\frac{1}{2}, \frac{1}{2} ; \frac{5}{2} ; \frac{1}{s}\right)\right\} \\
& =-(2 / 3) \sqrt{s}{ }_{2} F_{1}\left(\frac{3}{2},-\frac{1}{2} ; \frac{5}{2} ; \frac{1}{s}\right) .
\end{aligned}
$$

Next we calculate the function $u_{p}(s)$ for real values of $p$ between zero and one. After carrying out the $x$ integration in (3.8) one obtains for $y_{0}=\infty$

$$
u_{p}(s)=\frac{1}{\pi} \int_{0}^{\infty} \mathrm{d} y y^{p}\left\{-\frac{4}{3}-y^{2}+\frac{1}{4} y\left(y^{2}+s\right) \log \frac{y^{2}+2 y+s}{y^{2}-2 y+s}\right\} .
$$

First we substitute in (A.4) $z=y \mathrm{e}^{\pi \mathrm{i}}$ and secondly $z=y \mathrm{e}^{-\pi \mathrm{i}}$, next we add up 
both expressions with the result

$$
\begin{aligned}
\sin (p \pi) u_{p}(s)= & \frac{1}{2 \pi \mathrm{i}} \int_{\Gamma_{1}+\Gamma_{2}} \mathrm{~d} z z^{p} \\
& \times\left\{-\frac{4}{3}-z^{2}-\frac{1}{4} z\left(z^{2}+s\right) \log \frac{z^{2}-2 z+s}{z^{2}+2 z+s}\right\},
\end{aligned}
$$

where the contour $\Gamma_{1}$ is situated just above the branch cut of $z^{p}$, from $-\infty$ to 0 , and $\Gamma_{2}$ just below, from 0 to $-\infty$. Apart from the branch cut along the negative real $z$-axis, the integrand in (A.5) has two branch cuts along line segments from $z_{1}$ to $z_{3}$ and $z_{2}$ to $z_{4}$ respectively, where $z_{1}, \ldots, z_{4}$ are logarithmic branch points, with $z_{1,2}=1 \pm \mathrm{i} \sqrt{s-1}$ and $z_{3,4}=-1 \pm \mathrm{i} \sqrt{s-1}$. If we define $\Gamma_{3}$ and $\Gamma_{4}$ as closed contours around the upper and lower logarithmic branch cut respectively, both in the positive sense, we obtain, due to the behaviour of the integrand for $|z| \rightarrow \infty$, the following result

$$
\begin{aligned}
\sin (p \pi) u_{p}(s)= & \frac{-1}{2 \pi \mathrm{i}} \int_{\Gamma_{3}} \mathrm{~d} z z^{p} \frac{1}{4} z\left(z^{2}+s\right) \log \frac{z-z_{1}}{z-z_{3}} \\
& +\frac{-1}{2 \pi \mathrm{i}} \int_{\Gamma_{4}} \mathrm{~d} z z^{p} \frac{1}{4} z\left(z^{2}+s\right) \log \frac{z-z_{2}}{z-z_{4}} .
\end{aligned}
$$

Next we substitute $z=\mathrm{i} \sqrt{s-1}+x \pm \mathrm{i} \epsilon$ for $\Gamma_{3}$ and $z=-\mathrm{i} \sqrt{s-1}+x \pm \mathrm{i} \epsilon$ for $\Gamma_{4}$, where $\epsilon \rightarrow 0^{+}$, and $x$ runs from -1 to +1 . The upper signs have to be taken along the upper sides of the branch cuts, the lower signs along the lower sides. This yields

$$
\sin (p \pi) u_{p}(s)=\frac{1}{2} \sin \frac{p \pi}{2} \int_{-1}^{+1} \mathrm{~d} x\left\{(\sqrt{s-1}+\mathrm{i} x)^{p+3}-s(\sqrt{s-1}+\mathrm{i} x)^{p+1}\right\}
$$

which gives the final result

$$
\begin{array}{r}
u_{p}(s)=\frac{s^{(p+4) / 2}}{2 \cos (p \pi / 2)}\left[\frac{1}{p+4} \sin \left\{(p+4) \tan ^{-1} \frac{1}{\sqrt{s-1}}\right\}\right. \\
\left.-\frac{1}{p+2} \sin \left\{(p+2) \tan ^{-1} \frac{1}{\sqrt{s-1}}\right\}\right] .
\end{array}
$$

It is again convenient to have an expression for $u_{p}(s)$ in terms of hypergeometric functions, which can be obtained from the relation

$$
{ }_{2} F_{1}\left(\frac{p+1}{2},-\frac{p-1}{2} ; \frac{3}{2} ;(\sin z)^{2}\right)=\frac{\sin (p z)}{p \sin z}
$$


with the choice $\tan z=1 / \sqrt{s-1}$.

By using properties of contiguous functions ${ }^{9}$ ) one finds

$$
u_{p}(s)=-\frac{(p+3) s^{(p+1) / 2}}{3 \cos (p \pi / 2)}{ }_{2} F_{1}\left(\frac{p+5}{2},-\frac{p+1}{2} ; \frac{5}{2} ; \frac{1}{s}\right) .
$$

The function $v\left(y_{0}, \tau\right)$ follows from (3.23) and (3.7). The $s$-integral is performed by contour integration, so that

$$
v\left(y_{0}, \tau\right)=\frac{1}{\pi} \int_{0}^{y_{0}} \mathrm{~d} y y^{2} \mathrm{e}^{-y^{2} \tau} \int_{-1}^{+1} \mathrm{~d} x\left(1-x^{2}\right) \mathrm{e}^{2 x y \tau}-\frac{4}{3 \pi} y_{0} \delta(\tau) .
$$

The second term is omitted for $\tau \neq 0$. The limit for $y_{0} \rightarrow \infty$ of the first term is denoted by $v(\tau)$. The correction is estimated as

$$
v\left(y_{0}, \tau\right)=v(\tau)+\mathcal{O}\left(\mathrm{e}^{-y_{0}^{2} \tau}\right) .
$$

The $x$-integral in (A.8) can be represented in terms of the generalized hypergeometric function ${ }_{0} F_{1}{ }^{12}$ )

$$
\int_{-1}^{+1} \mathrm{~d} x\left(1-x^{2}\right) \mathrm{e}^{2 u x}=\frac{4}{3}{ }_{0} F_{1}\left(\frac{5}{2} ; u^{2}\right) .
$$

Thus

$$
v(\tau)=\frac{4}{3 \pi} \int_{0}^{\infty} \mathrm{d} y y^{2} \mathrm{e}^{-y^{2} \tau}{ }_{0} F_{1}\left(\frac{5}{2}, y^{2} \tau^{2}\right) .
$$

The integral takes the form of a Laplace transform after substitution of $y=\sqrt{t} / \tau$. The result is

$$
v(\tau)=\frac{1}{3 \sqrt{\pi}} \tau^{-3 / 2}{ }_{1} F_{1}\left(\frac{3}{2} ; \frac{5}{2} ; \tau\right) .
$$

The function $v_{p}\left(y_{0}, \tau\right)$ with $0<p<1$ follows from (3.24) and (3.8). Performing the $s$-integral yields

$$
\begin{aligned}
v_{p}\left(y_{0}, \tau\right)= & \frac{\tau}{\pi} \int_{0}^{y_{0}} \mathrm{~d} y y^{p+4} \mathrm{e}^{-y^{2} \tau} \\
& \times \int_{-1}^{+1} \mathrm{~d} x\left(1-x^{2}\right) \mathrm{e}^{2 x y \tau}-\frac{4}{3 \pi} \frac{y_{0}^{p+1}}{p+1} \delta(\tau) .
\end{aligned}
$$

For $\tau \neq 0$ and $y_{0} \rightarrow \infty$ we have

$$
v_{p}\left(y_{0}, \tau\right)=v_{p}(\tau)+\mathcal{O}\left(\mathrm{e}^{-y_{0}^{2} \tau}\right),
$$


where $v_{p}(\tau)$ is the limit of $v_{p}\left(y_{0}, \tau\right)$ for $y_{0} \rightarrow \infty$. Using (A.10) yields

$$
v_{p}(\tau)=\frac{4 \tau}{3 \pi} \int_{0}^{\infty} \mathrm{d} y y^{p+4} \mathrm{e}^{-y^{2} \tau}{ }_{0} F_{1}\left(\frac{5}{2} ; y^{2} \tau^{2}\right) .
$$

Substitution of $y=\sqrt{t} / \tau$ yields a Laplace transform, thus

$$
v_{p}(\tau)=\frac{2}{3 \pi} \Gamma\left(\frac{5+p}{2}\right) \tau^{-(3+p) / 2}{ }_{1} F_{1}\left(\frac{5+p}{2} ; \frac{5}{2} ; \tau\right) \text {. }
$$

\section{Appendix B}

Here we calculate the functions $g(\rho)$ and $g_{p}(\rho)$ defined in (3.52) and (3.53). The function $g(\rho)$ can be obtained from the following Fourier sine transform

$$
I(\alpha, \beta, y) \equiv \int_{0}^{\infty} \mathrm{d} x \sin (x y) x \mathrm{e}^{-\alpha x^{2}}{ }_{1} F_{1}\left(\beta ; \frac{3}{2} ; x^{2}\right),
$$

which we will calculate first, for complex values of $\beta$ with $\operatorname{Re} \beta<3 / 2$ and real values of $\alpha$ larger than one. In order to calculate (B.1) we need the following integral representation valid for $\operatorname{Re} \beta<3 / 2^{9}$ )

$$
\begin{aligned}
{ }_{1} F_{1}\left(\beta ; \frac{3}{2} ; x^{2}\right) & =\mathrm{e}^{x^{2}}{ }_{1} F_{1}\left(\frac{3}{2}-\beta ; \frac{3}{2} ;-x^{2}\right) \\
& =\frac{\Gamma(3 / 2) \Gamma(1-\beta)}{\Gamma(3 / 2-\beta) 2 \pi \mathrm{i}} \int_{0}^{(1+)} \mathrm{d} u u^{1 / 2-\beta}(u-1)^{\beta-1} \mathrm{e}^{(1-u) x^{2}} .
\end{aligned}
$$

The contour of integration is a loop starting (and ending) at $u=0$ and encircling 1 once in the positive sense. After substitution of (B.2) into (B.1) the $x$ integration can be carried out with the result

$$
I(\alpha, \beta, y)=\frac{1}{4} \sqrt{\pi} y \frac{\Gamma(3 / 2) \Gamma(1-\beta)}{\Gamma(3 / 2-\beta) 2 \pi \mathrm{i}} \int_{0}^{(1+)} \mathrm{d} u u^{1 / 2-\beta} v^{-3 / 2} \mathrm{e}^{-y^{2} /(4 v)},
$$

where $1 / v=\alpha-1+u$. The substitution $u=(\alpha-1) t /(\alpha-t)$, leaves the contour invariant, so that

$$
\begin{aligned}
I(\alpha, \beta, y)= & \frac{1}{4} \sqrt{\pi} y \mathrm{e}^{-y^{2} /(4 \alpha)} \frac{\alpha^{\beta-3 / 2}}{(\alpha-1)^{\beta}} \frac{\Gamma(3 / 2) \Gamma(1-\beta)}{\Gamma(3 / 2-\beta) 2 \pi \mathrm{i}} \\
& \times \int_{0}^{(1+)} \mathrm{d} t t^{1 / 2-\beta}(t-1)^{\beta-1} \exp \left\{-\frac{y^{2}}{4 \alpha(\alpha-1)}(1-t)\right\} .
\end{aligned}
$$


Using the integral representation (B.2) again gives finally

$$
I(\alpha, \beta, y)=\frac{1}{4} \sqrt{\pi} \frac{\alpha^{\beta-3 / 2}}{(\alpha-1)^{\beta}} y \mathrm{e}^{-y^{2} /(4 \alpha)}{ }_{1} F_{1}\left(\beta ; \frac{3}{2} ; \frac{-y^{2}}{4 \alpha(\alpha-1)}\right) .
$$

The result (B.5) is in fact valid for all complex $\beta$, as can be shown by using a different integral representation for ${ }_{1} F_{1}$. For our purposes, $\operatorname{Re} \beta<3 / 2$ is sufficient.

The function $g(\rho)$ can be calculated from the result (B.5) and from (3.52). By inserting for $w\left(\delta x^{2}\right)$ the expression (3.38a) and applying the property

$$
\frac{2}{3} y_{1} F_{1}\left(-\frac{1}{2} ; \frac{5}{2} ; y\right)={ }_{1} F_{1}\left(-\frac{1}{2} ; \frac{3}{2} ; y\right)-{ }_{1} F_{1}\left(-\frac{3}{2} ; \frac{3}{2} ; y\right)
$$

we arrive at the result

$$
\begin{aligned}
g(\rho)= & -2\left\{\frac{1-\delta}{\pi \delta}\right\}^{1 / 2}\left\{{ }_{1} F_{1}\left(-\frac{1}{2} ; \frac{3}{2} ;-\frac{\delta \rho^{2}}{4(1-\delta)}\right)\right. \\
& \left.-(1-\delta)_{1} F_{1}\left(-\frac{3}{2} ; \frac{3}{2} ;-\frac{\delta \rho^{2}}{4(1-\delta)}\right)\right\} .
\end{aligned}
$$

The previous method fails in the calculation of $g_{p}(\rho)$, and we have not been able to calculate $g_{p}(\rho)$ in closed form.

Here we will derive a series expansion in powers of $\rho$, which converges absolutely for all values of $\rho$, and an asymptotic expansion for large values of $\rho$. From (3.53) and (3.39) we have

$$
\begin{aligned}
g_{p}(\rho)= & -\frac{32 \Gamma((5+p) / 2) \delta^{(1-p) / 2} \mathrm{e}^{\rho^{2 / 4}}}{3 \pi^{3 / 2}\left(1-p^{2}\right) \rho} \\
& \times \int_{0}^{\infty} \mathrm{d} x \sin (x \rho) x^{3} \mathrm{e}^{-x^{2}}{ }_{2} F_{2}\left(\frac{5+p}{2},-\frac{1+p}{2}, \frac{3-p}{2}, \frac{5}{2} ; \delta x^{2}\right) .
\end{aligned}
$$

We insert the absolutely convergent expansion for ${ }_{2} F_{2}$ in (B.8), and integrate term by term using ${ }^{13}$ )

$$
\int_{0}^{\infty} \mathrm{d} x \sin (x \rho) \mathrm{e}^{-x^{2}} x^{2 m+3}=\frac{1}{2} \Gamma(5 / 2)(5 / 2)_{m} \rho \mathrm{e}^{-\rho^{2} / 4}{ }_{1} F_{1}\left(-m-1 ; \frac{3}{2} ; \rho^{2} / 4\right),
$$

where ${ }_{1} F_{1}$ is a polynomial of degree $(m+1)$ in $\rho^{2}$. The result is a hypergeometric series in two variables, $\rho^{2}$ and $\delta$, where $\delta$ is a given quantity smaller than 1 .

The most convenient representation for our purpose is

$$
g_{p}(\rho)=-\frac{4 \Gamma((5+p) / 2) \delta^{(1-p) / 2}}{\pi\left(1-p^{2}\right)} \sum_{m=0}^{\infty} \frac{\left(\frac{5+p}{2}\right)_{m}\left(-\frac{1+p}{2}\right)_{m}}{\left(\frac{3-p}{2}\right)_{m}\left(\frac{3}{2}\right)_{m} m !}
$$




$$
\times{ }_{2} F_{1}\left(m+\frac{5+p}{2}, m-\frac{1+p}{2} ; m+\frac{3-p}{2} ; \delta\right)\left\{1-\frac{\rho^{2}}{4 m+6}\right\}\left\{\frac{-\delta \rho^{2}}{4}\right\}^{m} .
$$

The asymptotic expansion of $g_{p}(\rho)$ can also be obtained from (B.8), if we extend in (B.8) the $x$ integral from $-\infty$ to $+\infty$, write $\sin (x \rho)=\operatorname{Im} \mathrm{e}^{\mathrm{i} x \rho}$ and substitute $x=z+\frac{1}{2} \mathrm{i} \rho$. Because ${ }_{2} F_{2}$ is an analytic function everywhere in the complex $z$-plane, the integration path may be shifted such that $-\infty<z<+\infty$. For fixed $\rho$ we may expand $\left(z+\frac{1}{2} \mathrm{i} \rho\right)^{3}{ }_{2} F_{2}\left(\ldots ; \delta\left(z+\frac{1}{2} \mathrm{i} \rho\right)^{2}\right)$, occurring in the resulting expression, in powers of $z$ and perform the $z$ integrals. This yields an expansion of the form

$$
\begin{aligned}
g_{p}(\rho)= & \frac{2 \Gamma((5+p) / 2) \delta^{(1-p) / 2}}{3 \pi\left(1-p^{2}\right) \rho}\left\{\sum_{m=0}^{\infty} \frac{(-)^{m}}{m !}\left(\frac{\partial}{\partial \rho}\right)^{2 m}\right\} \\
& \times \rho^{3}{ }_{2} F_{2}\left(\frac{5+p}{2},-\frac{1+p}{2} ; \frac{3-p}{2}, \frac{5}{2} ; \frac{-\delta \rho^{2}}{4}\right) .
\end{aligned}
$$

From the asymptotic expansion of ${ }_{2} F_{2}$ as given in ref. 10 one can deduce the asymptotic expansion of $g_{p}(\rho)$ and we give only the first four terms

$$
\begin{aligned}
g_{p}(\rho) & =\frac{\delta}{2(4+p) \cos (\pi p / 2) \rho} \sum_{m=0}^{3} \frac{(-)^{m}}{m !}\left(\frac{\partial}{\partial \rho}\right)^{2 m} \rho^{4+p}+\mathcal{O}\left(\rho^{-2-p}\right) \\
& =\frac{\delta}{2(4+p) \cos (\pi p / 2)} \rho^{3+p}\left\{1+\mathcal{O}\left(\rho^{-2}\right)\right\}
\end{aligned}
$$

\section{References}

1) J.W. Dufty and J.A. McLennan, Phys. Rev. A9 (1974) 1266.

2) T. Keyes and I. Oppenheim, Physica 70 (1973) 100.

3) I.M. de Schepper, H. van Beijeren and M.H. Ernst, Physica 75 (1974) 1.

4) W.W. Wood, Fundamental problems in Statistical Mechanics III, E.G.D. Cohen ed. (NorthHolland Publ. Co., Amsterdam, 1975).

5) R. Zwanzig, J. Chem. Phys. 40 (1964) 2527.

6) R. Zwanzig, Lectures in Theoretical Physics III, W.E. Brittin, B.W. Downs and J. Downs eds. (Interscience, New York, 1961).

7) M.H. Ernst, E.H. Hauge and J.M.J. van Leeuwen, J. Stat. Phys. 15 (1976) 7.

8) M.H. Ernst and J.R. Dorfman, J. Stat. Phys. 12 (1975) 311.

9) A. Erdelyi, W. Magnus, F. Oberhettinger and F.G. Tricomi, Higher Transcendental Functions I (McGraw-Hill, New York, 1953).

10) Y.L. Luke, The Special Functions and their Approximations (Academic Press, New York, 1969).

11) D. Bedeaux and P. Mazur, Physica 73 (1974) 431.

12) M. Abramowitz and I.A. Stegun, Handbook of Mathematical Functions (Dover Publ., New York, 1970).

13) A. Erdelyi, W. Magnus, F. Oberhettinger and F.G. Tricomi, Tables of Integral Transforms (McGraw-Hill, New York, 1954).

14) Y. Pomeau and P. Résibois, Physics Reports 19C (1975) 63.

15) I.M. de Schepper and M.H. Ernst, Generalized hydrodynamics for the diffusion process, thesis, University of Nijmegen (1975). 